\title{
26. Oscillatory Property of Certain Non-linear Ordinary Differential Equations
}

\author{
By Hiroshi ONOSE \\ Department of Mathematics, Ibaraki University \\ (Comm. by Zyoiti Suetuna, M. J. A., March 12, 1968)
}

1. Statement of theorems. Recently, Kartsatos [2] proved that certain differential equations of the form

$$
x^{\prime \prime}+f(t) g\left(x, x^{\prime}\right)=0 \quad \text { or } \quad x^{(2 n)}+f(t) g(x)=0
$$

can have only oscillatory solutions. Looking into the proofs in [2] closely, we see that the argument used there may be applied equally well to equations of the following more general form:

$$
x^{(2 n)}+f(t) g\left(x, x^{\prime}, \cdots, x^{(2 n-1)}\right)=0 .
$$

We shall prove in this paper the following theorems, where all functions considered are real-valued and continuous on their domains.

Theorem 1. Suppose that the differential equation (1) satisfies the following hypotheses:

$(\alpha) \quad f$ is a positive function defined on the interval $I=\left[t_{0},+\infty\right)$ with $t_{0} \geqslant 0$ and $\int_{t_{0}}^{+\infty} f(t) d t=+\infty$;

( $\beta) \quad g$ is defined on $R^{2 n}$; $\operatorname{sgn} g\left(x_{1}, x_{2}, \cdots, x_{2 n}\right)=\operatorname{sgn} x_{1}$ for any $\left(x_{1}\right.$, $\left.x_{2}, \cdots, x_{2 n}\right) \in R^{2 n}$; and $g\left(\lambda x_{1}, \lambda x_{2}, \cdots, \lambda x_{2 n}\right)=\lambda^{2 p+1} g\left(x_{1}, x_{2}, \cdots, x_{2 n}\right)$ for any $\left(x_{1}, x_{2}, \cdots, x_{2 n}\right) \in R^{2 n}$, any $\lambda \in R$ and some non-negative integer $p$. Then, every solution of (1) on the interval $I$ is oscillatory.

Theorem 2. Suppose that the equation (1) satisfies $(\alpha)$ and the following:

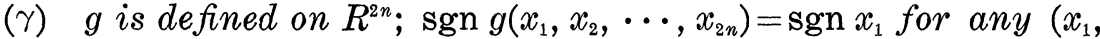
$\left.x_{2}, \cdots, x_{2 n}\right) \in R^{2 n} ; g\left(-x_{1},-x_{2}, \cdots,-x_{2 n}\right)=-g\left(x_{1}, x_{2}, \cdots, x_{2 n}\right)$ for any $\left(x_{1}, x_{2}, \cdots, x_{2 n}\right) \in R^{2 n}$; and for any $2 \leqslant k \leqslant 2 n-1$ and any $c \geqslant 0$, the function $g\left(x_{1}, x_{2}, \cdots, x_{2 n}\right)$ has a definite limit $G(k, c)$, which is positive or $+\infty$, as $x_{1} \rightarrow+\infty, \cdots, x_{k-1} \rightarrow+\infty, x_{k} \rightarrow c, x_{k+1} \rightarrow 0, \cdots, x_{2 n} \rightarrow 0$. Then, every solution of (1) on I is oscillatory.

We would like to remark that Kartsatos [2] proved Theorem 1 in the case $n=1$ and Theorem 2 when the function $g$ depends only on the variable $x_{1}$.

The author is indebted to Professor K. Nakashima for kind help and to Professor S. Sugiyama for pointing out errors in the first draft of this paper.

2. Proof of theorems. First we shall prove the following elementary but useful

Lemma. Let $\varphi$ be a 2n-times continuously differentiable func- 
tion defined on the interval $I=\left[t_{0},+\infty\right)$. If $\varphi>0$ and $\varphi^{(2 n)}<0$ on the interval $I$, then

$$
\lim _{t \rightarrow+\infty} \frac{\varphi^{(k)}(t)}{\varphi(t)}=0 \quad \text { for } 1 \leqslant k \leqslant 2 n-1 .
$$

Proof of lemma. As $\varphi^{(2 n)}<0$ on the interval $I, \varphi^{(2 n-1)}$ is decreasing on $I$, so that $\varphi^{(2 n-1)}(t)$ has a limit, finite or $-\infty$, as $t \rightarrow+\infty$. We denote this limit by $\varphi^{(2 n-1)}(\infty)$. We shall use similar notations in what follows.

Case 1: If $\varphi^{(2 n-1)}(\infty)<0$, then we see easily by integration that $\varphi^{(2 n-2)}(t) \rightarrow-\infty$ as $t \rightarrow+\infty$, and by the same argument as above $\varphi^{(k)}(t) \rightarrow-\infty$ as $t \rightarrow+\infty$ for $1 \leqslant k \leqslant 2 n-3$. And finally $\varphi(t) \rightarrow-\infty$ as $t \rightarrow+\infty$, which contradicts the hypothesis $\varphi>0$ on $I$.

Case 2: If $\varphi^{(2 n-1)}(\infty)>0$, then $\varphi^{(2 n-2)}(t) \rightarrow+\infty$ as $t \rightarrow+\infty$ and so $\varphi^{(k)}(t) \rightarrow+\infty$ as $t \rightarrow+\infty$ for $0 \leqslant k \leqslant 2 n-2$. In this case, we have $\varphi^{(k)}(t) / \varphi(t) \rightarrow 0$ as $t \rightarrow+\infty$ for $1 \leqslant k \leqslant 2 n-1$. In fact, this is trivial for $k=2 n-1$, because $\varphi^{(2 n-1)}(\infty)$ is finite. For $1 \leqslant k \leqslant 2 n-2$, it can be shown by using l'Hospital's rule.

Case 3: If $\varphi^{(2 n-1)}(\infty)=0$, then $\varphi^{(2 n-1)}>0$ on $I$ and therefore $\varphi^{(2 n-2)}$ is increasing on $I$. Thus, $\varphi^{(2 n-2)}(\infty)$ exists and is finite or $+\infty$. If $\varphi^{(2 n-2)}(\infty)<0$, then $\varphi^{(k)}(t) \rightarrow-\infty$ for $0 \leqslant k \leqslant 2 n-3$ and we get a contradiction. If $\varphi^{(2 n-2)}(\infty)>0$, then $\varphi^{(k)}(t) \rightarrow+\infty$ as $t \rightarrow+\infty$ for $0 \leqslant k \leqslant 2 n-3$. In this case, $\varphi^{(k)}(t) / \varphi(t) \rightarrow 0$ as $t \rightarrow+\infty$ by the above mentioned arguments, so that the lemma is true. If $\varphi^{(2 n-2)}(\infty)=0$, then $\varphi^{(2 n-2)}<0$ on $I$ and so $\varphi^{(2 n-3)}$ is decreasing on $I$. Here we may also have three cases: $\varphi^{(2 n-3)}(\infty)>0, \varphi^{(2 n-3)}(\infty)<0$ or $\varphi^{(2 n-3)}(\infty)=0$. In the first case, the lemma is true. The second case never happens, because it will lead to a contradiction. So we have only to dispose of the third case. Then, $\varphi^{(2 n-3)}>0$ on $I$ and we again have to examine the limit $\varphi^{(2 n-4)}(\infty)$.

Repeating similar arguments, we shall have only the following case that remains to be unsettled:

$$
(-1)^{k} \varphi^{(k)}<0 \text { and } \varphi^{(k)}(\infty)=0
$$

for $1 \leqslant k \leqslant 2 n-1$.

In this case, $\varphi$ is increasing. As $\varphi>0$ by hypothesis, we have $\varphi(\infty)>0$ and therefore $\varphi^{(k)}(t) / \varphi(t) \rightarrow \varphi^{(k)}(\infty) / \varphi(\infty)=0$ as $t \rightarrow+\infty$ for $1 \leqslant k \leqslant 2 n-1$. Hence our lemma is completely proved.

Q.E.D.

In the above proof, we have shown the following

Corollary. If $\varphi>0$ and $\varphi^{(2 n)}<0$ on $I$, then each $\varphi^{(k)}$ with $0 \leqslant k \leqslant 2 n-1$ has a definite limit as $t \rightarrow+\infty$. If we denote by $\varphi^{(k)}(\infty)$ these limits, then there happen only the following cases: $\varphi(\infty)=\cdots=\varphi^{(k-1)}(\infty)=+\infty, \varphi^{(k)}(\infty)=c \geqslant 0, \varphi^{(k+1)}(\infty)=\cdots=\varphi^{(2 n-1)}(\infty)=0$ with $0 \leqslant k \leqslant 2 n-1$, where $c>0$ in case $k=0$.

Proof of Theorem 1. Assume on the contrary that there exists a solution $x(t)$ of the equation (1) which does not oscillate on 
some interval $\left[t_{1},+\infty\right)$ with $t_{1} \geqslant t_{0}$. This solution $x$ can be supposed to be positive on the above interval. By (1), we have $x^{(2 n)}=-f(t)$ $\times g\left(x, x^{\prime}, \cdots, x^{(2 n-1)}\right)$ and so our hypothese simply that $x^{(2 n)}<0$ for all $t \geqslant t_{1}$. Using the lemma, we have

$$
\lim _{t \rightarrow+\infty} \frac{x^{(k)}(t)}{x(t)}=0 \quad \text { for } 1 \leqslant k \leqslant 2 n-1 .
$$

So, for any $\varepsilon>0$, there exists $T \geqslant t_{0}$ such that

$$
\left|g\left(1, \frac{x^{\prime}}{x}, \cdots, \frac{x^{(2 n-1)}}{x}\right)-g(1,0, \cdots, 0)\right|<\varepsilon \quad \text { for } t \geqslant T .
$$

As we have sgn $g(1,0, \cdots, 0)=\operatorname{sgn} 1$, so $g(1,0, \cdots, 0)>0$ and therefore we may assume $0<\varepsilon<g(1,0, \cdots, 0)$. As we see from the proof of the lemma, both $x^{\prime}$ and $x^{(2 n-1)}$ are non-negative for sufficiently large $t$. Thus we may assume that $x(t)>c_{0}, x^{\prime}(t) \geqslant 0, x^{(2 n-1)}(t) \geqslant 0$ and $x^{(2 n)}(t)<0$ for all $t \geqslant t_{2}$, where $t_{2} \geqslant \max \left(t_{1}, T\right)$ and $c_{0}$ is some positive constant.

If we put $y=x^{(2 n-1)} / x$, then we have

$$
y^{\prime}(t)=\frac{x^{(2 n)}(t)}{x(t)}-\frac{x^{\prime}(t) x^{(2 n-1)}(t)}{x(t)^{2}} \leqslant \frac{x^{(2 n)}(t)}{x(t)}
$$

for $t \geqslant t_{2}$. Integrating this inequality over the interval $\left[t_{2}, t\right]$ and using the equation (1), we have

$$
\begin{aligned}
\frac{x^{(2 n-1)}(t)}{x(t)} & -\frac{x^{(2 n-1)}\left(t_{2}\right)}{x\left(t_{2}\right)} \leqslant-\int_{t_{2}}^{t} \frac{f(s) g\left(x, x^{\prime}, \cdots, x^{(2 n-1)}\right)}{x(s)} d s \\
& =-\int_{t_{2}}^{t} f(s) x^{2 p}(s) g\left(1, \frac{x^{\prime}}{x}, \cdots, \frac{x^{(2 n-1)}}{x}\right) d s \\
& \leqslant-[g(1,0, \cdots, 0)-\varepsilon] c_{0}^{2 p} \int_{t_{2}}^{t} f(s) d s,
\end{aligned}
$$

which implies a contradiction; in fact, the last member tends to $-\infty$ as $t \rightarrow+\infty$, while the first member remains bounded.

Q.E.D.

Proof of Theorem 2. Let $x(t)$ be a non-oscillatory solution of the equation (1), which is assumed to be positive on $\left[t_{1},+\infty\right)$. The function $x^{(2 n-1)}(t)$ is decreasing for $t \geqslant t_{1}$, because of our hypotheses. By integrating the equation (1) from $t_{1}$ to $t$, we have

$$
-x^{(2 n-1)}(t)+x^{(2 n-1)}\left(t_{1}\right)=\int_{t_{1}}^{t} f(s) g\left(x, x^{\prime}, \cdots, x^{(2 n-1)}\right) d s .
$$

As it follows from Corollary in this section that $x^{(2 n-1)}(t) \geqslant 0$ for $t \geqslant t_{1}$, we have

$$
x^{(2 n-1)}\left(t_{1}\right) \geqslant \int_{t_{1}}^{t} f(s) g\left(x, x^{\prime}, \cdots, x^{(2 n-1)}\right) d s \quad \text { for } t \geqslant t_{1} .
$$

By the proof of the lemma, $x$ is increasing on the interval $\left[t_{1},+\infty\right)$ and, by the corollary, we have the following two cases, which we shall take care of one by one.

$$
\text { Case 1: } \quad x^{(m)}(\infty)= \begin{cases}0 & \text { for } k+1 \leqslant m \leqslant 2 n-1 \\ c & \text { for } m=k \\ +\infty & \text { for } 0 \leqslant m \leqslant k-1,\end{cases}
$$

where $0<k \leqslant 2 n-1$ and $c \geqslant 0$. Then, our assumption implies that 


$$
\lim _{t \rightarrow+\infty} g\left(x, x^{\prime}, \cdots, x^{(2 n-1)}\right)=G(k, c)>\varepsilon
$$

for some positive $\varepsilon$. So there exists a $t_{2} \geqslant t_{1}$ such that $g\left(x, x^{\prime}, \cdots\right.$, $\left.x^{(2 n-1)}\right)>\varepsilon$ for all $t \geqslant t_{2}$. It is obvious that the inequality (2) remains valid if we replace $t_{1}$ by $t_{2}$. Thus we obtain

Clearly this leads to a contradiction.

$$
x^{(2 n-1)}\left(t_{2}\right) \geqslant \varepsilon \int_{t_{2}}^{t} f(s) d s
$$

Case 2:

$$
x^{(m)}(\infty)= \begin{cases}0 & \text { for } 1 \leqslant m \leqslant 2 n-1 \\ c>0 & \text { for } m=0 .\end{cases}
$$

Then, given a positive number $\varepsilon<g(c, 0, \cdots, 0)$, there exists a $t_{2} \geqslant t_{1}$ such that $g(c, 0, \cdots, 0)-\varepsilon<g\left(x, x^{\prime}, \cdots, x^{(2 n-1)}\right)$ for all $t \geqslant t_{2}$ and we find from (2)

$$
x^{(2 n-1)}\left(t_{2}\right) \geqslant \int_{t_{2}}^{t} f(s) g\left(x, x^{\prime}, \cdots, x^{(2 n-1)}\right) d s \geqslant[g(c, 0, \cdots, 0)-\varepsilon] \int_{t_{2}}^{t} f(s) d s
$$

for all $t \geqslant t_{2}$, which again leads to a contradiction. $\quad$ Q.E.D.

3. Remarks. By considering the equation $x^{(3)}+x=0$, we see that it may be impossible to replace $2 n$ by $2 n+1$ in Theorems 1 and 2 .

As Kartsatos [2] has pointed out, we may improve the conditions in our theorems. For example, the homogeneity of $g$ in Theorem 1 may be assumed only for positive $\lambda$ and $p$ may be any non-negative number. But we prefer here to the brevity of exposition at the expense of generality.

Recently, Bhatia [1] and Tomastik [3] also have shown oscillatory properties for some second-order differential equations. But their hypotheses are somewhat different from ours.

\section{References}

[1] N. B. Bhatia: An oscillation theorem. Notices Amer. Math. Soc., 13, 243 (1966).

[2] A. G. Kartsatos: Some theorems on oscillations of certain non-linear secondorder differential equations. Arch. der Math., 18, 425-429 (1967).

[3] E. C. Tomastik: Oscillation of a non-linear second order differential equation. SIAM J. Appl. Math., 15, 1275-1277 (1966). 